\title{
Simulações Computacionais das Propriedades do Grafeno utilizando Processamento em GPUs
}

\author{
Gabriel Ortolan Fiano (IC) e Ana L. C. Pereira (PQ)
}

\begin{abstract}
Resumo
O grafeno apresenta propriedades únicas, com enorme potencial para aplicações, e o grupo vem estudando propriedades eletrônicas e efeitos de desordem neste material. $O$ objetivo desse projeto foi direcionado para o estudo dos ambientes computacionais em que são realizadas as simulações numéricas, testando-se o ambiente GPU do CENAPAD-SP. Foram estudados os tempos de processamento, assim como boas praticas de programação para que o tempo de execução das rotinas de programação seja o menor possível.
\end{abstract}

Palavras Chave: Grafeno, GPU, Problemas de Autovalores.

\section{Introdução}

O grafeno possui uma combinação única de propriedades e grande potencial para diversas aplicações ${ }^{1}$. No primeiro ano do projeto $^{2}$ estudamos dispositivos eletrônicos baseados em grafeno, analisando as consequências causadas por diferentes tipos de desordens nas propriedades desse revolucionário material, através de simulações numéricas que demandam clusters de alto desempenho. O objetivo do presente projeto foi começar a utilizar o ambiente GPU (Graphics Processing Unit) do Centro Nacional de Processamento de Alto Desempenho (CENAPAD-SP), comparando-se as vantagens e desvantagens deste ambiente em relação aos outros ambientes (CPUs) que o grupo já utilizava. O processamento com GPUs vem ganhando destaque nos últimos anos ao acelerar a capacidade para trabalhos complexos em diversas áreas, solucionando problemas que demandariam tempo muito maior em $\mathrm{CPUs}^{3}$.

\section{Resultados e Discussão}

Para as simulações foi considerado o modelo de rede tight-binding para monocamadas de grafeno, como modelo base. Foi utilizado o software Matlab, rodando no ambiente GPU do CENAPADSP. Foram analisados os tempos de execução dos programas, que envolvem a diagonalização da matriz Hamiltoniana do sistema para diferentes cenários: somente autovalores, ou também os autovetores, matrizes reais ou matrizes complexas. A desordem foi incluída fazendo-se variar as energias dos átomos ou considerandose vacâncias. O tempo de processamento aumenta exponencialmente com a dimensão da matriz a ser diagonalizada, como mostra a Figura 1 , sendo que essa dimensão corresponde ao número total de átomos na rede considerada.

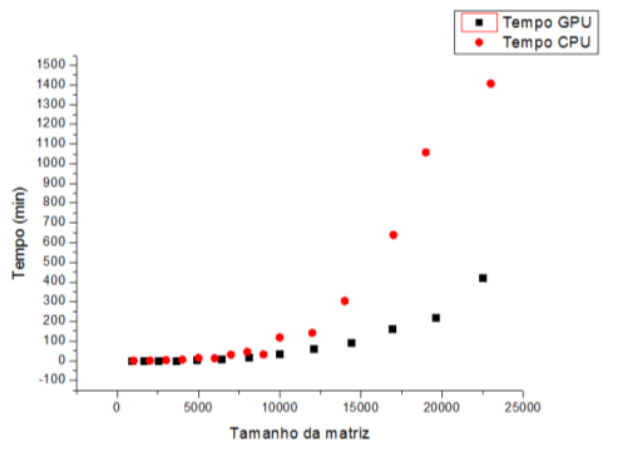

Figura 1. Comparação entre os tempos de processamento das simulações nos ambientes CPU e GPU do CENAPAD-SP, em função da dimensão da matriz diagonalizada.

\section{Conclusões}

Foi possível concluir que o sistema GPU é muito mais eficiente para a diagonalização de matrizes do que o sistema CPU, mapeando em detalhes os tempos de processamento. Outros integrantes do grupo começaram também a utilizar este sistema, beneficiando-se dos testes realizados neste projeto. Vale ressaltar que a obtenção de autovalores e autovetores de uma matriz não é utilizada apenas nos estudos de grafeno, mas também em diversas outras áreas de pesquisa.

\section{Agradecimentos}

Agradeço a dedicação, apoio e companheirismo da prof ${ }^{a}$ Ana Luiza e todos os integrantes do grupo durante os dois anos de pesquisa. Agradeço também ao CNPq pela bolsa PIBIC e ao apoio do suporte técnico do CENAPAD-SP.

\footnotetext{
${ }^{1}$ Castro Neto A. H., Guinea F., Peres N. M. R., Novoselov K. S. and Geim A. K., Rev. Mod. Phys., 2009, 81, 109-162.

Fiano G. O. e Pereira A. L. C., resumo PIBIC 2014: http://www.prp.rei.unicamp.br/pibic/congressos/xxiicongresso/cdrom /FSCOMMAND/pdfN/345.pdf

${ }^{3}$ Cardoso, E. A. "Análise comparativa de algoritmos NP-Completo executados em CPU e GPU utilizando CUDA”. 2012.
} 